\title{
1 Elements of Algebra
}

In this first chapter we introduce the algebraic structures that will be used in the following chapters. Definitions are enriched with lots of examples and figures for better understanding. We will only deal with commutative rings and number fields and therefore every reference to a ring or a field implies a commutative ring and a number field respectively.

\subsection{Domains}

In this section we investigate some basic properties of domains that are used throughout the textbook. Starting with the algebraic structure of the commutative ring and adding properties to it, we establish the algebraic structure of the field.

Definition A commutative ring $R$ is called an integral domain if for any two elements $a, b \in R, a \cdot b=0$ implies that $a=0$ or $b=0$.

The definition of integral domain is necessary in order to have a domain where divisibility can occur. As we know the set of $n \times n$ invertible matrices, where the identity element of the ring is the identity matrix and the zero element of the ring is the zero matrix, form a commutative ring. The product of two matrices $A, B$ might be the zero matrix while neither of $A$ and $B$ are zero matrices. For instance

$$
\left[\begin{array}{ll}
0 & 0 \\
0 & 2
\end{array}\right] \cdot\left[\begin{array}{ll}
2 & 0 \\
0 & 0
\end{array}\right]=\left[\begin{array}{ll}
0 & 0 \\
0 & 0
\end{array}\right]
$$

Thus, the ring of $n \times n$ diagonal matrices is not an integral domain, even though it is a commutative ring. All fields, the ring of rational integers $\mathbb{Z}$ and all the subrings of integral domains are integral domains.

Proposition 1.1.1. If $R$ is an integral domain then $R[x]$ is also an integral domain.

Proof. In order to prove that $R[x]$ is an integral domain it is equivalent to prove that for any two elements

$$
f(x)=a_{n} x^{n}+\cdots+a_{1} x+a_{0}, a_{n} \neq 0
$$

and

$$
g(x)=b_{m} x^{m}+\cdots+b_{1} x+b_{0}, b_{m} \neq 0
$$

if $f(x) g(x)=0$ then $f(x)=0$ or $g(x)=0$. The product $f(x) g(x)$ is

$$
f(x) g(x)=a_{n} b_{m} x^{n+m}+\cdots+\left(a_{1} b_{0}+a_{0} b_{1}\right) x+a_{0} b_{0}=0 .
$$

Thus $a_{n} b_{m}=0$, which is a contradiction. 
Divisibility in commutative rings give rise to interesting properties for the elements of those rings. Those properties inspire the following definitions. Let $R$ be an integral domain. An element $u \in R$ is called $u$ it if there is $u^{-1} \in R$ such that $u \cdot u^{-1}=1$ and is called $n$-th root of unity if $u^{n}=1$ for some $n$ positive integer. The element $u^{-1}$ is called an invertible element of $u$. An element $r \in R$ is called irreducible if there are no non-unit elements $a, b$ of $R$ such that $a \cdot b=r$. An element $p \in R$ is called prime if for any $a, b$ of $R$ such that $p \mid a b$, then $p \mid a$ or $p \mid b$. The definition of the prime element is equivalent to the condition that $p$ is prime if the principal ideal $(p)$ is a prime ideal. Two elements $r_{1}$ and $r_{2}$ of $R$ are called associates if there is a unit $u \in R$ such that $r_{1}=u r_{2}$.

Proposition 1.1.2. The prime elements of an integral domain are irreducible.

Proof. Let $p$ be a prime element of an integral domain $R$. Then, for any $a, b \in R$ such that $p=a b$ we have that $p \mid a$ or $p \mid b$. Assume, without loss of generality, that $p \mid a$. Then there is $c \in R$ such that $a=p c$. Hence,

$$
p=a b \Rightarrow p=p c b \Rightarrow c b=1 \Rightarrow b \text { is a unit }
$$

and therefore $p$ is irreducible.

Definition An integral domain $R$ in which every non-zero element $a$ can be written uniquely with respect to a unit as a product of irreducible elements of $R$ is called a unique factorization domain.

This means that if we have an element $a$ of a unique factorization domain $R$ that can be factorized in two ways, for instance

$$
a=b_{1}^{q_{1}} \cdots b_{n}^{q_{n}}=c_{1}^{r_{1}} \cdots c_{m}^{r_{m}}
$$

where $b_{i}$ and $c_{j}$ are irreducible elements of $R$ and $q_{i}$ and $r_{j}$ are rational integers, then necessarily $n=m$ and each irreducible factor $b_{i}$ is equal up to a unit to exactly one irreducible factor $c_{j}$. The definition of the unique factorization domain arises from the generalization of the Fundamental Theorem of Arithmetic which states that every positive integer except 1 is either a prime or it can be written uniquely as a product of prime elements. A historical survey of the Fundamental Theorem of Arithmetic can be found in (Agargün et al., 2001).

Now, let $R$ be the polynomial ring $\mathbb{Z}[\sqrt{-5}]$ which is the ring that consists of the elements $a+b \sqrt{-5}$ for any $a, b \in \mathbb{Z}$. The element $9 \in \mathbb{Z}[\sqrt{-5}]$ can be written in two ways. That is

$$
9=3 \cdot 3=(2+\sqrt{-5})(2-\sqrt{-5}) .
$$

Since 3 does not differ from either $(2+\sqrt{-5})$ or $(2-\sqrt{-5})$ by more than a unit, we conclude that 9 has two representations with irreducible elements and therefore $\mathbb{Z}[\sqrt{-5}]$ 
is not a unique factorization domain. On the other hand the set of the Gaussian integers $\mathbb{Z}[\sqrt{-1}]=\mathbb{Z}[i]$ is a unique factorization domain and the irreducible Gaussian integers are exactly the rational integer primes which are not a sum of two rational integers and the elements of the form $a \pm b i$ such that $a^{2}+b^{2}$ is prime. In addition if $R$ is a unique factorization domain then so is $R[x]$.

Proposition 1.1.3. Every irreducible element of a unique factorization domain is prime.

Proof. Let $r$ be an irreducible element of the unique factorization domain $R$. Assume that $r \mid a b$ for some $a, b \in R$. Then there is a $c \in R$ such that $a b=c r$. Since $R$ is a unique factorization domain, $a$ and $b$ can be factorized uniquely into irreducible elements as follows

$$
a_{1} \cdots a_{n} b_{1} \cdots b_{m}=c r .
$$

Thus $r$ must be associate to a factor of either $a$ or $b$. Therefore $r \mid a$ or $r \mid b$ which implies that $r$ is prime.

Definition An integral domain in which every ideal is principal is called a principal ideal domain.

This means that every ideal of $R$ can be generated by a single element. If $a$ and $b$ are elements of the same principal ideal domain then there is a $d$ such that the principal ideal $(d)$ generated by $d$ is equal to the ideal $(a, b)$ generated by $a$ and $b$. If $a$ and $b$ have no common divisors then every element of the $R$ can be represented as $a r_{1}+b r_{2}$ for some $r_{1}$ and $r_{2}$ of $R$.

Every field $K$ is a principal ideal domain since its trivial ideal (0) is unique and therefore at the same time maximal. Next we will show that the ring of integers $\mathbb{Z}$ and the polynomial ring $K[x]$ are also principal ideal domains.

Proposition 1.1.4. The ring of integers $\mathbb{Z}$ is a principal ideal domain.

Proof. Let $I$ be a non zero ideal of $\mathbb{Z}$ and $a$ the smallest positive integer of $I$. According to the euclidean division for any element $b \in I$ it holds that

$$
b=\pi a+v
$$

where $0 \leq v<a$. Since $b, \pi a$ are elements of $I$ it implies that $v \in I$ but since $v<a$ and $a$ is the smallest positive integer of $I$ we deduce that $v=0$. Thus, $b=\pi a$ and therefore $I$ is equal to the principal ideal $(a)$.

Proposition 1.1.5. For any field $K$, the polynomial ring $K[x]$ is a principal ideal domain. 
Proof. Let $I$ be a non-zero ideal of $K[x]$ and $f(x)$ an element of $I$ having the minimal degree of all elements of $K[x]$. Assume that there is a $g(x) \in I$ and that

$$
g(x)=\pi(x) f(x)+v(x)
$$

where $0 \leq \operatorname{deg} v(x)<\operatorname{deg} f$. Since $g(x)$ and $\pi(x) f(x)$ are elements of $I$ we deduce that $v(x)$ is also an element of $I$. But $f(x)$ is an element of minimum degree and $0 \leq \operatorname{deg} v(x)<\operatorname{deg} f$, so $v(x)=0, g(x)=\pi(x) f(x)$ and therefore $I=(f(x))$.

Examples of domains that are not principal ideal domains are the bivariate ring $K[x, y]$ and the polynomial ring $\mathbb{Z}[x]$. For example the ideal generated by $(x, y)$ is not a principal ideal of $K[x, y]$ and the ideal $(x, n)$ generated by the two elements $x$ and $n \in \mathbb{Z}$ where $n \geq 2$ is not a principal ideal of $\mathbb{Z}[x]$.

Proposition 1.1.6. If $R$ is a principal ideal domain, then every prime ideal of $R$ is maximal.

Proof. Assume that $(a)$ is a non-maximal prime ideal of $R$. Then there is a principal ideal $(b)$ such that $(a) \subsetneq(b) \subsetneq R$. Thus, $a \in(b)$ and there is $c \in R$ such that $a=b c$. Since $b \notin(a)$ it implies that $c \in(a)$. Thus there is $d \in R$ such that $c=d a$ and therefore

$$
a=b c \Rightarrow a=b d a \Rightarrow b d=1 \text {. }
$$

Hence, 1 belongs to $(b)$ which means that $(b)=R$. This is a contradiction since we assumed that $(b) \subsetneq R$.

Proposition 1.1.7. Every principal ideal domain is a unique factorization domain.

Proof. In order to prove that a principal ideal domain $R$ is a unique factorization domain it is equivalent to show that every non-unit element $a$ of $R$ has a factorization into irreducible factors with respect to a unit. Initially assume that none of the factors of $a$ is irreducible. Let $a_{1} \in R$ such that $a_{1} \mid a$. Then $a_{1}$ does not have any irreducible factors. Inductively, we can create an infinite sequence of non-irreducible factors $\cdots a_{2}\left|a_{1}, a_{1}\right| a$ such that

$$
\text { (a) } \subsetneq\left(a_{1}\right) \subsetneq\left(a_{2}\right) \subsetneq \cdots
$$

Now, assume that $I$ is generated by $a, a_{1}, a_{2}, \ldots$ Since $R$ is a principal ideal domain, there is a $b \in R$ such that

$$
b=\sum_{i=0}^{n} r_{i} a_{i}, \quad r_{i} \in R, a_{0}=a .
$$

Thus $b \in\left(a_{n}\right)$ and $(b) \subset\left(a_{n}\right)$. On the other hand we have that

$$
\left(a_{n}\right) \subsetneq\left(a, a_{1}, a_{2}, \ldots\right)=I .
$$


This is a contradiction with the assumption that $a$ was irreducible, and therefore we have proved that every element $a$ of $R$ has at least one irreducible factor.

Suppose now that $a=a_{1} r_{1}$ where $r_{1}$ is the irreducible factor of $a$. If $a_{1}$ is a unit then $a$ is irreducible. If $a_{1}$ is not a unit then there is an irreducible factor $r_{2}$ and some $a_{2} \in R$ such that $a_{1}=a_{2} r_{2}$. If $a_{2}$ is a unit then $a=a_{2} r_{1} r_{2}$ is a factorization of $a$ into irreducible factors. This procedure could be continued indefinitely, and therefore $a$ has an irreducible factor.

The following theorem is very enlightening since it provide us a sufficient and necessary condition for a unique factorization theorem to be a principal ideal domain

Theorem 1.1.8. A unique factorization domain $R$ is a principal ideal domain if and only if every prime ideal of $R$ is maximal.

Proof. We will show that if every prime ideal of $R$ is maximal then $R$ is a principal ideal domain, since the converse is obvious by 1.1.7.

Let $I$ be a non-trivial ideal of $R$ and $a, b \in R$ where

$$
a=a_{1} \cdots a_{n}
$$

and

$$
b=b_{1} \cdots b_{m}
$$

are the decompositions of $a$ and $b$ respectively into irreducible elements such that $a_{i} \neq b_{j}$ for any $i$ and $j$ with respect to the units of $R$. Since $I$ is prime, at least one factor from $p_{i}$ and $b_{j}$ belong to $I$. Without loss of generality suppose that $p_{1}, q_{1} \in I$. Since $p_{1}, q_{1}$ are irreducibles, by 1.1 .3 we get that $\left(p_{1}\right)$ and $\left(q_{1}\right)$ are primes and maximal by our assumption. This is a contradiction since $p_{1} \neq q_{1}$ and therefore it holds that

$$
\left(p_{1}\right) \subsetneq\left(p_{1}, q_{1}\right) \subset I \subsetneq R
$$

and

$$
\left(q_{1}\right) \subsetneq\left(p_{1}, q_{1}\right) \subset I \subsetneq R .
$$

Now let $\mathcal{J}$ be the set of non-principal ideals of $R$ and assume that $\mathcal{J}$ is not an empty set. Let

$$
I_{1} \subset I_{2} \subset \cdots
$$

and

$$
I=\bigcup\left(I_{i}\right)
$$

If $I$ is a principal ideal ( $a$ ), then $a \in I_{i}$ for some $i$ and therefore $I_{i}=(a)$. This is a contradiction and therefore $I \in \mathcal{J}$. By Zorn's lemma (see (Vereshchagin and Shen, 2002) or (Komjáth and Totik, 2006)), we get that there is $I$ a maximal ideal of $\mathcal{J}$. If $I$ is not a prime ideal, then there exist $a, b \in R$ such that $a b \in I$ and $a, b \notin I$. Then

$$
I \subsetneq(I, a)=(c)
$$


and

$$
I \subsetneq(I, b)=d
$$

for some $c, d \in R$. Hence

$$
I=(I, a b)=(I, a)(I, b)=(c)(d)=(c d),
$$

which is a contradiction. There is no I satisfying those conditions which implies that $\mathcal{J}$ is an empty set.

Next we will investigate the properties of the so called Noetherian rings. We will only investigate the Noetherian rings that are commutative. So when we refer to a Noetherian ring we will always mean a commutative Noetherian ring.

Definition A ring in which every ascending sequence of ideals

$$
I_{0} \subset I_{1} \subset I_{2} \subset \cdots
$$

there exists an $n$ such that

$$
I_{0} \subset I_{1} \subset I_{2} \subset \cdots \subset I_{n}=I_{n+1}=\cdots
$$

is called a Noetherian ring or Noetherian domain.

Proposition 1.1.9. Let $R$ be a ring. The following sentences are equivalent

(i) For every ascending sequence of ideals

$$
I_{1} \subset I_{2} \subset I_{3} \subset \cdots
$$

there is an index $n$ such that $I_{n}=I_{n+1}=\cdots$.

(ii) Every non-empty set of ideals of $R$ has a maximal ideal.

(iii) Every ideal of $R$ is finitely generated.

Proof. (i) $\rightarrow$ (ii). Let $I_{1}$ be a non-maximal ideal of a non-empty set of ideals J. Thus, there is an ideal $I_{2} \in \mathcal{J}$ such that $I_{1} \subsetneq I_{2}$. If $I_{2}$ is not maximal then there is an ideal $I_{3} \in \mathcal{J}$ such that $I_{2} \subsetneq I_{3}$. In this way, we can construct an infinite sequence

$$
I_{1} \subset I_{2} \subset I_{3} \subset \cdots
$$

which by the hypothesis after $n$ ideals it becomes eventually constant. Therefore $I_{n}$ is the maximal ideal of $\mathcal{J}$.

(ii) $\rightarrow$ (iii). Let $I_{1}$ be an ideal of $R$ and $\mathcal{J}$ the set of ideals of $R$ that are generated by finitely many elements of $I_{1}$. J is not an empty set since $(0) \in \mathcal{J}$. Now, assume that there is element $I_{2}=\left(a_{1}, \ldots, a_{n}\right)$ in $\mathcal{J}$ which is maximal and not equal to $I_{1}$. Then there is $a \in I_{1}$ such that $a \notin I_{2}$ which implies that there is $I_{3}=\left(a_{1}, \ldots, a_{n}, a\right)$ such that $I_{2} \subsetneq I_{3}$. This is a contradiction since we assumed that $I_{2}$ is a maximal set of $\mathcal{J}$ and therefore such an $a$ cannot exist. Thus $I_{2}=I_{1}$ and therefore $I_{1}$ is finitely generated. 
(iii) $\rightarrow$ (i). Let

$$
I_{1} \subset I_{2} \subset I_{3} \subset \cdots
$$

be an ascending sequence of ideals in $R$ and define the ideal

$$
I=\bigcup_{i=1}^{\infty} I_{i} .
$$

Suppose that $I$ is generated by $\left(a_{1}, \ldots, a_{n}\right)$. All $a_{i}$ belong to at least one of those $I_{i}$. Assume $I_{n}$ includes all the others $I_{i}$ and since all $I_{i}$ belong to an ascending sequence we deduce that $I_{n}=I$ which means that the ascending sequence becomes constant after $I_{n}$.

Since every ideal of a principal ideal is generated by a single element we deduce the following corollary.

Corollary 1.1.10. Every principal ideal domain is a Noetherian domain.

We can easily verify that $\mathbb{Z}$ is a Noetherian ring as well as the polynomial $\operatorname{ring} K[x]$ for a given field $K$. But in the case when we have a ring $R$, the proof that $R[x]$ is Noetherian is non-trivial. The theorem that proves this assumption is called Hilbert's basis theorem. Inductively it can be also shown that if $R$ is Noetherian then $R\left[x_{1}, \ldots, x_{n}\right]$ is as well. On the other hand, the polynomial rings of infinite variables $R\left[x_{1}, x_{2}, \ldots\right]$ are not Notherian rings since the infinite ascending sequence

$$
\left(x_{1}\right) \subset\left(x_{1}, x_{2}\right) \subset \cdots
$$

never becomes constant.

Proposition 1.1.11. If a ring $R$ is Noetherian and every irreducible element of $R$ is prime, then $R$ is a unique factorization domain.

Proof. Assume that the set $\mathcal{J}$ of ideals that are generated by each one of the elements of $R$ that are not units and that can not be factorized into irreducible elements of $R$ is not an empty set. Then, since $R$ is Noetherian there is a maximal ideal $I=(a)$ where $a$ is not irreducible and therefore not prime. So $(a)$ is not a prime ideal which is a contradiction since every maximal ideal is prime. Hence, we deduce that all elements of $R$ can be written as a product of irreducible factors.

Let us try to prove the uniqueness. Let $a \in R$ such that

$$
a=b_{1} \cdots b_{n}=c_{1} \cdots c_{m}
$$

where $b_{i}$ and $c_{j}$ are primes, $n, m \in \mathbb{Z}$ and $n<m$. It holds that $b_{1}$ divides $c_{1} \cdots c_{m}$ and since $b_{1}$ is a prime, we can assume without loss of generality that $b_{1}$ divides $c_{1}$. 
Then $b_{1}=u_{1} c_{1}$ and since both $b_{1}$ and $c_{1}$ are irreducible we deduce that $u_{1}$ is a unit. Following the same procedure for all $b_{i}$ we obtain the relation

$$
b_{1} \cdots b_{n}=u_{1} \cdots u_{n} b_{1} \cdots b_{n} c_{n+1} \cdots c_{m}
$$

which implies that

$$
1=u_{1} \cdots u_{n} c_{n+1} \cdots c_{m} .
$$

Since $u_{i}$ are units we deduce that $c_{n+1}, \ldots, c_{m}$ are also units which is a contradiction. So $n=m$ and $b_{i}$ are equal $c_{i}$ with respect to the unit

$$
u=u_{1} \cdots u_{n},
$$

where $u_{i}$ units of $R$.

Definition An integral domain that

(i) is Noetherian,

(ii) is integrally closed (see p.13) and

(iii) every nonzero prime ideal of it is maximal

is called Dedekind domain.

An immediate corollary of the definition of the Dedekind domain and proposition 1.1.8 follows.

Corollary 1.1.12. A Dedekind domain $R$ that is a unique factorization domain is a principal ideal domain.

Now we have a better view of the main algebraic domains and the figure 1.1 that follows gives us a minimal representation of how those domains are connected to each other.

\subsection{Rings and Fields}

A very interesting type of ring regarding the properties that it carries is the local ring.

Definition A ring that contains a unique maximal ideal is called local ring.

Thus, all fields are local rings, since its unique trivial ideal (0) is at the same time maximal. An easy example to help understand the structure of a local ring is the field of rational numbers with the elements with denominators that are generated by a natural number removed. For example, the ring that contains the elements of $\mathbb{Q}$ minus the elements with denominator divisible by 5 is a local ring. A very interesting and useful property of local ring is provided by the proposition that follows. By $R-I$ we denote the set

$$
R-I=\{r \in R \mid r \notin I\} .
$$




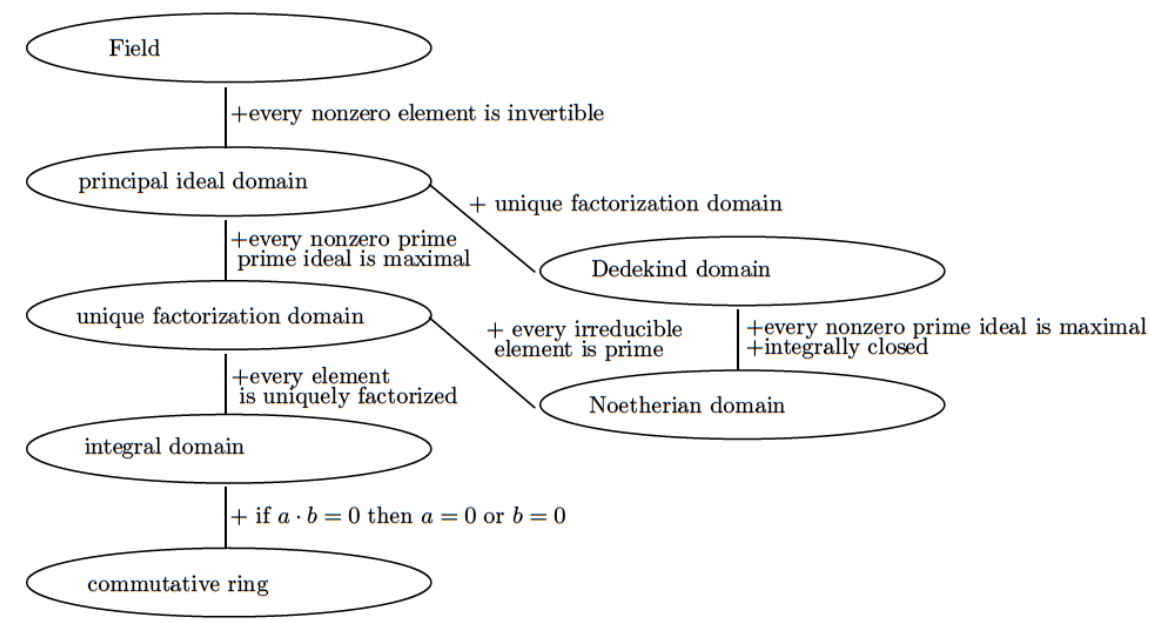

Figure 1.1: Domains

Proposition 1.2.1. If $R$ is a local ring and $I$ its maximal ideal then the subset $R-I$ of $R$ is precisely the set of units of $R$.

Proof. Suppose that there is an $a \in R-I$ such that $a$ is not a unit, then there is another ring containing $a$ and therefore there is another maximal ideal besides $I$. Conversely, if $I^{\prime}$ is another maximal ideal, then there exists $a \in I^{\prime}$ and $a \notin I$. If $a$ was a unit then $I^{\prime}$ would be $R$. Therefore, $a$ is not a unit.

It is obvious that for any field $K$ with maximal ideal $\{0\}$ the elements $K-\{0\}$ are all invertible. Consider the local ring which contains the elements of $\mathbb{Q}$ less the elements with denominator divisible by 5 . The maximal ideal, are the elements with numerator divisible by 5 . All the other elements are invertible.

Now let $R$ and $S$ be two rings with $S \subset R$. We remind to the reader that an element $r \in R$ is called integral over $S$ if there are $s_{i} \in S$ such that

$$
r^{n}+s_{n-1} r^{n-1}+\cdots+s_{1} r+s_{0}=0 .
$$

The set of elements of $R$ that are integral over $S$ is called the integral closure of $S$ in $R$. In the case where the integral closure of $S$ in $R$ coincides with $R$, then we say that $R$ is integral over $S$ and $R$ is called extension ring of $S$.

The procedure where from a ring $S$ we obtain an extended ring $R$ is called ring extension. In order to extend a ring $S$ we attach an element $r$ that is not in $S$. Thus, the set

$$
R=S[r]=\left\{s_{0}+s_{1} r+\cdots+s_{n-1} r^{n-1} \mid s_{i} \in S, r \notin S\right\}
$$


is the extended ring of $S$ by attaching $r$. The same way we can attach finitely many elements. For instance, if in the ring of integers $\mathbb{Z}$ we attach $i$, we get the ring of Gaussian integers $\mathbb{Z}[i]$. The polynomial ring $S[x]$ is also a ring extension of $S$.

Definition The ring of all integral elements over $\mathbb{Z}$ of a field $K$ is called the ring of integers of $K$ and is denoted by $O_{K}$.

The ring of integers $O_{K}$ of $K$ have a basis which is called the integral basis of $K$. So, $O_{K}$ is a free $\mathbb{Z}$-module which means that if $\left\{a_{1}, \ldots, a_{n}\right\}$ is an integral basis of $K$ then

$$
O_{K}=\left\{z_{1} a_{1}+\cdots+z_{n} a_{n} \mid z_{i} \in \mathbb{Z}\right\} .
$$

For example the ring of integers of the rational field $\mathbb{Q}$ is $\mathbb{Z}$ and its basis is the trivial $\{1\}$.

Analogous definitions occur for fields.

Definition Let $L$ and $K$ be two fields with $K \subset L$. An element $y \in L$ is called algebraic over $K$ if there are $a_{i} \in K$ such that

$$
y^{n}+a_{n-1} y^{n-1}+\cdots+a_{1} y+a_{0}=0
$$

If the element is not algebraic, then it is called transcendental. If every element of $L$ is algebraic over $K$ then we say that $L$ is an algebraic extension of $K$.

For example $i \in \mathbb{C}$ is algebraic over $\mathbb{R}$ since $i$ is a root of $y^{2}+1$. Also, $\sqrt{2}$ is algebraic over $\mathbb{Q}$ since it is a root of $y^{2}-2$, while $\pi$ is not, since there is no polynomial with rational coefficients having $\pi$ as a root. The field extension of $L$ over $K$ is denoted by $L / K$. It is easy to verify that if $L / K$ and $M / L$ are algebraic extensions the so $M / K$ is.

Given a field $K$ we can create a larger field that contains $K$. If $a \notin K$, then by the field

$$
K(a)=\left\{\frac{f(a)}{g(a)} \mid f(x), g(x) \in K[x], g(x) \neq 0\right\} .
$$

For example, for $a=\sqrt{2}$ and $K=\mathbb{Q}$ we get the field

$$
\mathbb{Q}(\sqrt{2})=\{x+y \sqrt{2} \mid x, y \in \mathbb{Q}\} .
$$

This is a field extension and in particular the smallest extension of $\mathbb{Q}$ containing $\sqrt{2}$.

Definition A field $K$ is called algebraically closed if $K$ contains all the roots of all polynomials $K[x]$.

For instance, the field of rational numbers is not algebraically closed, since the roots of $x^{2}-2$ are not contained in $\mathbb{Q}$. On the other hand, the field of complex numbers is an algebraically closed field. 
Definition The minimal polynomial $p(x)$ of $a$ over a field $K$ is called separable if the number of its distinct roots are equal to $\operatorname{deg} p(x)$. In that case, the extension $K(a) / K$ is a separable extension.

For example the field extension $\mathbb{Q}(i) / \mathbb{Q}$ is separable since the minimal polynomial of $i$ is $x^{2}+1$ and it has two distinct roots, $i$ and $-i$. In particular, all field extensions of number fields are separable.

Definition If $L / K$ is a field extension, then the dimension of $L$ as a $K$-vector space is called the degree of $L$ over $K$ and is denoted by $[L: K]$ or $\operatorname{dim}_{K} L$. In the case when $K=\mathbb{Q}$ the degree of the extension $L / \mathbb{Q}$ is simply denoted by $\operatorname{dim} L$.

Therefore, the basis of $L$ over $K$ consists of $[L: K]$ elements. One of the basic properties of field dimension is that if $L / K$ and $M / L$ are two field extensions then

$$
[M: K]=[M: L] \cdot[L: K] .
$$

This property is proved easily using minimal polynomials.

The field extension can be characterised as a finite extension or an infinite extension depending to the dimension of its extension. If $[L: K]<\infty$ then $L / K$ is a finite extension, otherwise it is called infinite. The finite extensions are algebraic extensions. The extensions of degree 2 are often called quadratic extensions and the extensions of degree 3 are often called cubic extensions.

Definition The smallest field extension that contains two field extensions $L / K$ and $M / K$ is called compositum of $L$ and $M$.

The compositum of two fields $L$ and $M$ is usually denoted as $L M$.

For an integral domain $R$, define the set

$$
R^{\prime}=\left\{\left(a_{1}, a_{2}\right) \mid a_{1}, a_{2} \in R, a_{2} \neq 0\right\}
$$

and the equivalence relation

$$
\left(a_{1}, a_{2}\right) \sim\left(b_{1}, b_{2}\right) \Longleftrightarrow a_{1} b_{2}=a_{2} b_{1} .
$$

So, the equivalence classes that arise by the above equivalence relation we call them fractions and it holds that

$$
\frac{a_{1}}{a_{2}}=\frac{b_{1}}{b_{2}} \Longleftrightarrow\left(a_{1}, a_{2}\right) \sim\left(b_{1}, b_{2}\right) .
$$

The set of all fractions under the usual operations of multiplication and addition form a field which is called field of fractions of $R$. For example the field of fractions of the ring of integers is the field of rationals and the field of fractions of $K[x]$ for a given field $K$ is $K(x)$. 
Definition An integral domain whose integral closure in its field of fraction is itself is called an integrally closed domain.

Let $a_{1}, \ldots, a_{n}$ be elements of $\mathbb{R}^{n}$ which are linearly independent over $\mathbb{R}$. Then, $\left\{a_{1}, \ldots, a_{n}\right\}$ consists a basis for $\mathbb{R}^{n}$ as a vector space over $\mathbb{R}$. The set $\left\{a_{1}, \ldots, a_{n}\right\}$ forms a basis for a free $\mathbb{Z}$-module of rank $n$.

Definition The set

$$
\Lambda=\left\{\sum_{i=1}^{n} z_{i} a_{i} \mid z_{i} \in \mathbb{Z}\right\}
$$

where $a_{1}, \ldots, a_{n}$ is a basis for $\mathbb{R}^{n}$ is called a lattice.

Thus, every lattice in $\mathbb{R}^{n}$ consists of the elements that are generated by the elements of a basis of $\mathbb{R}^{n}$ and all their linear combinations with integer coefficients.

\subsection{Norm and Trace}

Two of the most interesting constructions that are associated to field extensions are norm and trace.

Definition In a field extension $L / K$ with $[L: K]=n$ the norm of an element $a \in L$ over $K$ is

$$
N_{L / K}(a)=\left(\prod_{i=1}^{n} a_{i}\right)^{[L: K(a)]}
$$

and the trace of an element $a$ is

$$
\operatorname{Tr}_{L / K}(a)=[L: K(a)] \sum_{i=1}^{n} a_{i}
$$

where $a_{i}$ are the conjugate roots of the minimal polynomial of $a$ over $K$.

In order to distinguish the cases were $K$ is the field of rationals the norm over a non trivial extension $K$ of $\mathbb{Q}$ is called relative norm of an element. Analogously, we refer to the trace over a non trivial extension $K$ of $\mathbb{Q}$ as relative trace of an element. Obviously, in case $a \in K$ then

$$
N_{L / K}(a)=a^{\operatorname{dim}_{K} L}
$$

and

$$
\operatorname{Tr}_{L / K}(a)=a \cdot \operatorname{dim}_{K} L
$$

For example, suppose that we have the extension $\mathbb{Q}(\sqrt{2}) / \mathbb{Q}$ and we need to compute the norm and the trace of $a=2-\sqrt{2}$. The minimal polynomial of $a$ is $x^{2}-4 x+2$ 
and $[\mathbb{Q}(\sqrt{2}) / \mathbb{Q}(a)]=1$. The other root of $x^{2}-4 x+2$ is $a_{2}=2+\sqrt{2}$. Thus

$$
N_{\mathbb{Q}(\sqrt{2}) / \mathbb{Q}}(a)=a_{1} \cdot a_{2}=8
$$

and

$$
\operatorname{Tr}_{\mathbb{Q}(\sqrt{2}) / \mathbb{Q}}(a)=a_{1}+a_{2}=4
$$

Some of the basic properties of norm and trace are arising from the following lemma.

Lemma 1.3.1. For any algebraic extension $L / K$ it holds that

(i) $N_{L / K}(a b)=N_{L / K}(a) \cdot N_{L / K}(b)$, for $a, b \in L$.

(ii) $N_{L / K}(k a)=k^{[L / K]} N_{L / K}(a)$, for $a \in L$ and $k \in K$.

(iii) $\operatorname{Tr}_{L / K}\left(k_{1} a+k_{2} b\right)=k_{1} \operatorname{Tr}_{L / K}(a)+k_{2} \operatorname{Tr}_{L / K}(b)$, for $a, b \in L$ and $k_{1}, k_{2} \in K$.

Proof. The proof of the lemma is an immediate application of the definitions of norm and trace.

A homogeneous form in $[L: K]$ variables based on the norm of the field extension $L / K$ is called norm form. That is, the map

$$
\begin{aligned}
N: L & \rightarrow K \\
\chi_{1} a_{1}+\cdots+x_{n} a_{n} & \rightarrow N_{L / K}\left(\chi_{1} a_{1}+\cdots+x_{n} a_{n}\right)
\end{aligned}
$$

where $\left\{a_{1}, \ldots, a_{n}\right\}$ is the basis of $L$ over $K$ and $x_{i}$ the variables. An equation of the form

$$
N_{L / K}\left(x_{1} a_{1}+\cdots+x_{n} a_{n}\right)=k
$$

is called the norm form equation and will see an efficient algorithm for solving those kind of equations in Chapter 7. Norm forms are useful for solving Diophantine equations, such as the Pell equations (Alvanos and Poulakis, 2009).

Let $L=\mathbb{Q}(a)$ be a separable extension, $p(x)$ the minimal polynomial of $a$ and $\left\{a_{1}, \ldots, a_{n}\right\}$ the set of all roots of $a$ in $\mathbb{C}$. The homomorphism

$$
\begin{aligned}
\sigma_{i}: L & \hookrightarrow \mathbb{C} \\
a & \mapsto a_{i}
\end{aligned}
$$

is called embedding of $L$. If $\sigma_{i}(a) \in \mathbb{R}$ then the embedding is called a real embedding, otherwise it is called a complex embedding. Since in a polynomial the complex roots appear in pairs, if $r$ is the number of real embeddings and $s$ the number of pairs of the complex embeddings then $r+2 s=n$.

In terms of embeddings if $L / K$ is a separable extension, then the trace of an element is

$$
\operatorname{Tr}_{L / K}(a)=\sum_{i=1}^{n} \sigma_{i}(a)
$$


and the norm of an element is

$$
N_{L / K}(a)=\prod_{i=1}^{n} \sigma_{i}(a) .
$$

Moreover, if the minimal polynomial of $a$ over $K$ is

$$
x^{r}+a_{r-1} x^{r-1}+\cdots+a_{1} x+a_{0}
$$

it can be proved that

$$
\operatorname{Tr}_{L / K}(a)=-\frac{n}{r} \cdot a_{r-1}
$$

and

$$
N_{L / K}(a)=(-1)^{n} a_{0}^{\frac{n}{r}} .
$$

Thus, the trace and the norm of an element $a$ of an extension $L / K$ is an element of $K$. In particular, if $K=\mathbb{Q}$ then $N(a)$ and $\operatorname{Tr}(a)$ are rational integers.

\subsection{Group of Units}

The units in a ring $R$ form under the operation of multiplication a group called the group of units or unit group of $R$ and is usually denoted by $U_{R}$. This group might be finite as in the case of the integers $\mathbb{Z}$ where the units are $\{-1,1\}$ or it can be infinite as in the case of the ring of integers of $\mathbb{Q}(\sqrt{2})$. It is easy to verify that $(3-2 \sqrt{2})^{s}$ for any $s \in \mathbb{N}$ is a unit of $O_{\mathbb{Q}(\sqrt{2})}$ and therefore $U_{\mathbb{Q}(\sqrt{2})}$ is an infinite group. In particular, if $O_{K}$ is the ring of integers of $K$, then by Dirichlet's Unit Theorem we get that the group of units of a ring of integers is a finitely generated abelian group.

Theorem 1.4.1 (Dirichlet's Unit Theorem). Let $K$ be a field, $r_{1}$ the number of real embeddings and $r_{2}$ the number of conjugated pairs of complex embeddings. Then, the group of units $U_{K}$ of $O_{K}$ is isomorphic to $Z \times \mathbb{Z}^{r}$ where $r=r_{1}+r_{2}-1$ and $Z$ is the finite cyclic group of the roots of unity in $O_{K}$.

Proof. Section 7.4 in (Jarvis, 2014).

This theorem provide us with the exact number of multiplicatively independent generators needed in order to compute the unit group of the ring of integers of a field. By the term independent generators we refer to the elements whose product equals 1 if and only if they are in power of 0 , i.e. $u_{1}, \ldots, u_{r}$ are linearly independent units of $O_{K}$ if and only if

$$
u_{1}^{k_{1}} \cdots u_{r}^{k_{r}}=1 \Longleftrightarrow k_{1}=\cdots=k_{r}=0 .
$$

So, every unit $u$ of $O_{K}$ can be represented as follows:

$$
u=\zeta u_{1}^{k_{1}} \cdots u_{r}^{k_{r}}
$$

where $k_{1}, \ldots, k_{r} \in \mathbb{Z}$ and $\zeta$ is a root of unity. 
Definition The finite cyclic group of the roots of unity in a ring of integers is called the torsion group while the group that is generated by the multiplicatively independent generators is called torsion free part of the group or just free part of the group. The set of the generators that generates the free part of the group is called fundamental set of units.

Proposition 1.4.2. Let a be an integral element of $O_{K}$

(i) $a$ is a unit $\Longleftrightarrow N(a)= \pm 1$

(ii) a is irreducible $\Longleftrightarrow N(a)$ is a prime rational integer

Proof. (i). Initially we will show that if $a$ is a unit then $N(a)= \pm 1$. So, if $a$ is a unit there is $b \in O_{K}$ such that $a b=1$. Thus,

$$
a b=1 \Rightarrow N(a b)=N(1) \Rightarrow N(a) N(b)=1
$$

and since $N(a)$ is a rational integer we deduce that $N(a)= \pm 1$. Conversely, if $N(a)= \pm 1$ then by definition of the norm

$$
a_{1} \cdots a_{n-1} a= \pm 1
$$

where $a_{1}, \ldots, a_{n-1}$ are the conjugates of $a$. Thus, $a$ is a unit with invertible element $a_{1} \cdots a_{n-1}$ or $-a_{1} \cdots a_{n-1}$.

(ii). Now, let $a$ be an integral element of $O_{K}$ with norm equal to a prime rational integer. Assume that there are $b, c \in O_{K}$ such that $a=b c$. By applying norm on both sides we get that $N(a)=N(b) N(c)$. Since $N(a)$ is prime we get that $N(b)= \pm 1$ or $N(c)= \pm 1$. Hence, $b$ or $c$ is a unit and therefore $a$ is irreducible. Conversely, assume that $a$ is irreducible and that $N(a)$ is not a prime rational integer. Then there is a unit $b$ and an integral element $c$ such that $a=b c$ and $N(a)=N(b) N(c)$ with $N(b) \neq \pm 1$, which is a contradiction according to (i).

The above proposition combined with the definition of relative norm gives the definition of relative units over a number field.

Definition An element $u \in L$ of a finite extension $L / K$ is called a relative unit over $K$ if and only if $N_{L / K}(u)=\zeta$, where $\zeta$ is root of unit in $K$. The group that consists of all relative units is called relative unit group.

It is of interest to compute the generators of a relative unit group in the field of algebra in general. We compute unit groups in Chapter 7 in order to compute norm form equations. 